Proyecciones Journal of Mathematics

Vol. 35, No 4, pp. 381-393, December 2016.

Universidad Católica del Norte

Antofagasta - Chile

\title{
On generalization of $K$-divergence, its order relation with $J$-divergence and related results
}

\author{
G. Farid \\ COMSATS Institute of Information Technology, Pakistan \\ Atiq Ur Rehman \\ COMSATS Institute of Information Technology, Pakistan \\ and \\ J. Pečarić \\ University Of Zagreb, Croatia \\ Received: May 2015. Accepted : September 2016
}

\begin{abstract}
In this paper, we give an order relation between J-divergence and generalized K-divergence. By using this order relation we give generalizations of the results related to an order relation between J-divergence and K-divergence given by J. Burbea and C. R. Rao. Also we construct class of $m$-exponentially convex functions introducing by nonnegative difference of new order relation.
\end{abstract}

Subjclass [2000] : 94A17, 26B25

Keywords : Convex functions, K-divergence, J-divergence, log-convexity, Cauchy means, exponentially convex functions. 


\section{Introduction}

The importance of suitable measures of distance between probability distributions arises because of the role they play in the problems of inference and discrimination. The concept of distance between two probability distributions was initially developed by Mahalanobis [9]. Since then various types of distance measures have been developed in the literature, see Burbea and Rao[3] and Rao [14]. Many of the currently used econometric tests, such as the likelihood ratio, the score and Wald tests, can in fact be shown to be in terms of appropriate distance measures. A concept closely related to the one of distance measures is that of divergence measures based on the idea of information-theoretic entropy first introduced in communication theory by Shannon [15] and later by Wiener [17] in Cybernetics. The origin of the term entropy, however, goes back to the work of Clausius [5] and Boltzman [2] in thermodynamics. Although it is well-known that the two concepts are related, here we consider Shannon's concept of information-theoretic entropy. (see, [16])

Let $I$ be an interval in $\mathbf{R}$ and $\mathbf{x}:=\left(x_{1}, \ldots, x_{n}\right), \mathbf{y}:=\left(x_{1}, \ldots, x_{n}\right) \in I^{n}$. For a given function $\phi$ defined on an interval $I$, the $\phi$ entropy of $\mathbf{x}$ is defined as follows: $\mathrm{H}_{n, \phi}(\mathbf{x})=-\sum_{i=1}^{n} \phi\left(x_{i}\right)$. For two vectors $\mathbf{x}$ and $\mathbf{y}$, the Jensen difference in term of $\phi$ entropy is defined as follows:

$$
J_{n, \phi}(\mathbf{x}, \mathbf{y})=H_{n, \phi}\left(\frac{\mathbf{x}+\mathbf{y}}{2}\right)-\frac{1}{2}\left[H_{n, \phi}(\mathbf{x})+H_{n, \phi}(\mathbf{y})\right] .
$$

Among several divergence measures defined in the statistical literature to reflect the fact that some probability distributions are closer together than other, and consequently, that it may be easier to distinguish between the distribution of one pair than between those of the others. An important measure of divergence is $J_{n, \phi}$ defined in (1.1) also known as $J$-divergence [4]. This divergence has some interesting properties (see [4] and [6, p. 16]). For applications of this divergence see $[8,13,14]$. Another important measure of divergence is $K$-divergence introduced by Burbea and Rao [4] . This measure is defined as:

$$
K_{n, \phi}(\mathbf{x}, \mathbf{y})=\sum_{i=1}^{n}\left(x_{i}-y_{i}\right)\left(\frac{\phi\left(x_{i}\right)}{x_{i}}-\frac{\phi\left(y_{i}\right)}{y_{i}}\right),
$$

where function $\phi$ is on an interval $I$ not containing zero such that $\frac{\phi(x)}{x}$ is increasing function and $\mathbf{x}, \mathbf{y} \in I^{n}$.

In [4] there is given an order relation between $J$-divergence and $K$-divergence measures. 
Theorem 1.1. For any $\mathrm{x}, \mathrm{y} \in \mathbf{R}_{+}^{n}$

$$
4 J_{n, \phi}(\mathbf{x}, \mathbf{y}) \geq K_{n, \phi}(\mathbf{x}, \mathbf{y})
$$

if and only if $\frac{\phi(x)}{x}$ is convex for $x \in \mathbf{R}_{+}$and that the equality occurs if and only if $\mathbf{x}=\mathbf{y}$.

In [1] we considered generalization of $K$-divergence (2.1) and proved its exponentially convexity, log-convexity by using a particular class of convex functions. Also mean value theorems are proved by using this generalization. In this paper we give an order relation between $J$-divergence and generalized $K$-divergence. Also results related to this new order relation are given which appear as generalizations of results given in $[1,7]$.

We construct class of $m$-exponentially convex functions introducing by non-negative difference of new order relation. It is noted that many classes related to convex functions are consequences of $m$-exponentially convex functions such as positive functions, log-convex functions and exponentially convex functions. Some new classes of relative convex functions are given in $[11]$.

\section{Main results}

A generalization of $K$-divergence is defined as follows:

Definition 2.1. [1] Let $I$ be an interval in $\mathbf{R}, \mathbf{x}, \mathbf{y} \in I^{n}$ and $\mathbf{p}:=\left(p_{1}, \ldots, p_{n}\right) \in$ $(0,1]^{n}$. Also let $f, h$ be real valued functions on interval $I$ such that $h(x) \neq 0$ for all $x \in I$. We define $T$-divergence, denoted by $T_{n, f}(\mathbf{x}, \mathbf{y} ; \mathbf{p})$ as follows:

$$
T_{n, f}(\mathbf{x}, \mathbf{y} ; \mathbf{p})=\sum_{i=1}^{n} p_{i}\left(h\left(x_{i}\right)-h\left(y_{i}\right)\right)\left(\frac{f\left(x_{i}\right)}{h\left(x_{i}\right)}-\frac{f\left(y_{i}\right)}{h\left(y_{i}\right)}\right) .
$$

Let us note that if $\frac{f}{h}$ is increasing functions on $I$, then $T_{n, f}(\mathbf{x}, \mathbf{y} ; \mathbf{p})$ is non-negative.

If $h$ is convex in Jensen sense then, in the following theorem we have an order relation between $J$-divergence and generalized $K$-divergence.

Theorem 2.2. Let $\mathbf{x}, \mathbf{y} \in I^{n}$, where $I$ is some interval in $\mathbf{R}$ and $h$ be positive $J$-convex function on $I$. If $\frac{f}{h}$ is $J$-convex function and $\mathbf{p} \in(0,1]^{n}$, then the following inequality holds

$$
4 J_{n, \phi}(\mathbf{x}, \mathbf{y}) \geq T_{n, f}(\mathbf{x}, \mathbf{y} ; \mathbf{p}),
$$

equality occurs if and only if $\mathbf{p}=\mathbf{1}$ and $\mathbf{x}=\mathbf{y}$. 
Proof. This statement is equivalent to the specialized statement with $n=1$, therefore we prove (2.2) for $n=1$.

As $\frac{f}{h}$ is J-convex and $h$ is positive therefore we have $2 \mathrm{~h}\left(\frac{x+y}{2}\right)\left(\frac{f(x)}{h(x)}+\frac{f(y)}{h(y)}\right)-$ $4 f\left(\frac{x+y}{2}\right) \geq 0$. Using J-convexity of function $h$ we get

$$
(\mathrm{h}(\mathrm{x})+\mathrm{h}(\mathrm{y}))\left(\frac{f(x)}{h(x)}+\frac{f(y)}{h(y)}\right)-4 f\left(\frac{x+y}{2}\right) \geq 0 .
$$

After rearranging terms and using that $0<p_{1} \leq 1$ we have $4\left(\frac{1}{2}(f(x)+f(y))-f\left(\frac{x+y}{2}\right)\right)-p_{1}(h(x)-h(y))\left(\frac{f(x)}{h(x)}-\frac{f(y)}{h(y)}\right) \geq 0$, that is $4 \mathrm{~J}_{1, \phi}(x, y) \geq T_{1, f}(x, y ; p)$

Remark 2.3. If we set $h(x)=x, p_{i}=1(i=1, . ., n)$ in (2.2) we get (1.3).

Now we give results related to difference of $J$-divergence and generalized $K$-divergence measures. Following lemma is important to give next results.

Lemma 2.4. Let $\frac{f}{h} \in C^{2}(I), I$ is compact interval in $\mathbf{R}$ and $h(x) \neq 0$ for all $x \in I$. Also let $m$ and $M$ be such that

$$
m \leq\left(\frac{f(x)}{h(x)}\right)^{\prime \prime} \leq M \forall x \in I .
$$

Consider the functions $\phi_{1}, \phi_{2}$ defined as

$$
\phi_{1}(x)=\frac{M x^{2} h(x)}{2}-f(x),
$$

and

$$
\phi_{2}(x)=f(x)-\frac{m x^{2} h(x)}{2},
$$

then $\frac{\phi_{i}}{h}$, for $i=1,2$, are convex on $I$.

Proof. Since

$$
\left(\frac{\phi_{1}(x)}{h(x)}\right)^{\prime \prime}=M-\left(\frac{f(x)}{h(x)}\right)^{\prime \prime} \geq 0, \forall x \in I,
$$

this gives $\frac{\phi_{1}}{h}$ is convex function on $I$. Similarly we have that $\frac{\phi_{2}}{h}$ is convex function on $I$ too.

For further generalized results in the following, we give teh definition of new generalized divergence measure. 
Definition 2.5. Let $I$ be an interval in $\mathbf{R}, \mathbf{x}, \mathbf{y} \in I^{n}$ and $\mathbf{p} \in(0,1]^{n}$. For real valued functions $f, h$ defined on $I$, we define, $D$-divergence $D_{n, f}(\mathbf{x}, \mathbf{y}, \mathbf{p})$ as:

$$
D_{n, f}(\mathbf{x}, \mathbf{y} ; \mathbf{p})=4 J_{n, f}(\mathbf{x}, \mathbf{y})-T_{n, f}(\mathbf{x}, \mathbf{y} ; \mathbf{p}) .
$$

If $h$ is positive convex in Jensen sense and $\frac{f}{h}$ is convex function in Jensen sense then the divergence defined in (2.4) is non-negative.

Theorem 2.6. Let $I$ be a compact interval in $\mathbf{R}, \mathbf{x}, \mathbf{y} \in I^{n}$ and $\mathbf{p} \in(0,1]^{n}$. If $\frac{f}{h} \in C^{2}(I)$ such that $h(x) \neq 0$ for all $x \in I$, then there exists $\xi \in I$ such that the following equality is valid

$$
\begin{gathered}
D_{n, f}(\mathbf{x}, \mathbf{y} ; \mathbf{p})=\frac{h^{2}(\xi) f^{\prime \prime}(\xi)-2 h(\xi) h^{\prime}(\xi) f^{\prime}(\xi)+f(\xi)\left(2 h^{\prime}(\xi)-h(\xi) h^{\prime \prime}(\xi)\right)}{h^{3}(\xi)} \times \\
\sum_{i=1}^{n}\left\{\left(x_{i}^{3}+y_{i}^{3}\right)-\frac{1}{4}\left(x_{i}+y_{i}\right)^{3}-\frac{p_{i}}{2}\left(h\left(x_{i}\right)-h\left(y_{i}\right)\right)\left(\frac{x_{i}^{3}}{h\left(x_{i}\right)}-\frac{y_{i}^{3}}{h\left(x_{i}\right)}\right)\right\} .
\end{gathered}
$$

Proof. As $\frac{f}{h} \in C^{2}(I), I$ is compact interval, we suppose that

$$
\min _{x \in I}\left(\frac{f(x)}{h(x)}\right)^{\prime \prime}=m \text { and } \max _{x \in I}\left(\frac{f(x)}{h(x)}\right)^{\prime \prime}=M .
$$

If we set $f=\phi_{1}$ in $(2.2)$ we have $\sum_{i=1}^{n}\left[4\left\{\frac{1}{2}\left(\phi_{1}\left(x_{i}\right)+\phi_{1}\left(y_{i}\right)\right)-\phi_{1}\left(\frac{x_{i}+y_{i}}{2}\right)\right\}\right.$ $\left.-p_{i}\left(h\left(x_{i}\right)-h\left(y_{i}\right)\right)\left(\frac{\phi_{1}\left(x_{i}\right)}{h\left(x_{i}\right)}-\frac{\phi_{1}\left(y_{i}\right)}{h\left(y_{i}\right)}\right)\right] \geq 0$.

Using definition of $\phi_{1}$ from Lemma 2.4 we get

$\sum_{i=1}^{n}\left[4\left\{\frac{1}{2}\left(M \frac{x_{i}^{3}}{2}+M \frac{y_{i}^{3}}{2}-f\left(x_{i}\right)-f\left(y_{i}\right)\right)-\frac{M}{2}\left(\frac{x_{i}+y_{i}}{2}\right)^{3}+f\left(\frac{x_{i}+y_{i}}{2}\right)\right\}\right.$ $\left[-p_{i}\left(h\left(x_{i}\right)-h\left(y_{i}\right)\right)\left(\frac{M}{2}\left(\frac{x_{i}^{3}}{h\left(x_{i}\right)}-\frac{y_{i}^{3}}{h\left(y_{i}\right)}\right)-\frac{f\left(x_{i}\right)}{h\left(x_{i}\right)}+\frac{f\left(y_{i}\right)}{h\left(y_{i}\right)}\right)\right] \geq 0$, which gives us the inequality

$D_{n, f}(\mathbf{x}, \mathbf{y} ; \mathbf{p}) \leq M \sum_{i=1}^{n}\left[\left(x_{i}^{3}+y_{i}^{3}\right)-\frac{1}{4}\left(x_{i}+y_{i}\right)^{3}-p_{i}\left(h\left(x_{i}\right)-h\left(y_{i}\right)\right)\left(\frac{x_{i}^{3}}{h\left(x_{i}\right)}-\frac{y_{i}^{3}}{h\left(y_{i}\right)}\right)\right]$.

Now if we set $f=\phi_{2}$ in (1.3) and use its definition from Lemma 2.4, we get

$D_{n, f}(\mathbf{x}, \mathbf{y} ; \mathbf{p}) \geq m \sum_{i=1}^{n}\left[\left(x_{i}^{3}+y_{i}^{3}\right)-\frac{1}{4}\left(x_{i}+y_{i}\right)^{3}-p_{i}\left(h\left(x_{i}\right)-h\left(y_{i}\right)\right)\left(\frac{x_{i}^{3}}{h\left(x_{i}\right)}-\frac{y_{i}^{3}}{h\left(y_{i}\right)}\right)\right]$.

By using the fact $m \leq\left(\frac{f}{h}\right)^{\prime \prime} \leq M$, inequalities (2.5) and (2.6), there exists $\xi \in I$ such that (2.6) holds. 
Corollary 2.7. If we put $h(x)=x$ and $p_{i}=1$, for $i=1, \ldots, n$ in (2.6), then we get [1, Theorem 2.3].

Theorem 2.8. Let $I$ be a compact interval in $\mathbf{R}, \mathbf{x}, \mathbf{y} \in I^{n}$ and $\mathbf{p} \in(0,1]^{n}$. If $\frac{f}{h}, \frac{g}{h} \in C^{2}(I)$ and $h(x) \neq 0$ for all $x \in I$, then there exists $\xi \in I$ such that the following equality is valid:

$$
\frac{D_{n, f}(\mathbf{x}, \mathbf{y} ; \mathbf{p})}{D_{n, g}(\mathbf{x}, \mathbf{y} ; \mathbf{p})}=\frac{h^{2}(\xi) f^{\prime \prime}(\xi)-2 h(\xi) h^{\prime}(\xi) f^{\prime}(\xi)+f(\xi)\left(2 h^{\prime}(\xi)-h(\xi) h^{\prime \prime}(\xi)\right)}{g^{2}(\xi) g^{\prime \prime}(\xi)-2 g(\xi) g^{\prime}(\xi) g^{\prime}(\xi)+g(\xi)\left(2 g^{\prime}(\xi)-g(\xi) g^{\prime \prime}(\xi)\right)}
$$

provided denominators are not zero.

Proof. Let we set $k=c_{1} f-c_{2} g$, where $c_{1}:=D_{n, g}(\mathbf{x}, \mathbf{y} ; \mathbf{p})$ and $c_{2}:=$ $D_{n, f}(\mathbf{x}, \mathbf{y} ; \mathbf{p})$. Then $\frac{k}{h} \in C^{2}(I)$ and using Theorem 2.6, with $f=k$, we have

$$
0=\left(c_{1} \frac{h^{2}(\xi) f^{\prime \prime}(\xi)-2 h(\xi) h^{\prime}(\xi) f^{\prime}(\xi)+f(\xi)\left(2 h^{\prime}(\xi)-h(\xi) h^{\prime \prime}(\xi)\right)}{h^{3}(\xi)}\right.
$$

$$
\left.-c_{2} \frac{h^{2}(\xi) g^{\prime \prime}(\xi)-2 h(\xi) h^{\prime}(\xi) g^{\prime}(\xi)+g(\xi)\left(2 h^{\prime}(\xi)-h(\xi) h^{\prime \prime}(\xi)\right)}{h^{3}(\xi)}\right) \times
$$

$\sum_{i=1}^{n}\left\{\left(x_{i}^{3}+y_{i}^{3}\right)-\frac{1}{4}\left(x_{i}+y_{i}\right)^{3}-\frac{p_{i}}{2}\left(h\left(x_{i}\right)-h\left(y_{i}\right)\right)\left(\frac{x_{i}^{3}}{h\left(x_{i}\right)}-\frac{y_{i}^{3}}{h\left(x_{i}\right)}\right)\right\}$.

From which we get

$$
\frac{c_{2}}{c_{1}}=\frac{h^{2}(\xi) f^{\prime \prime}(\xi)-2 h(\xi) h^{\prime}(\xi) f^{\prime}(\xi)+f(\xi)\left(2 h^{\prime}(\xi)-h(\xi) h^{\prime \prime}(\xi)\right)}{h^{2}(\xi) g^{\prime \prime}(\xi)-2 h(\xi) h^{\prime}(\xi) g^{\prime}(\xi)+g(\xi)\left(2 h^{\prime}(\xi)-h(\xi) h^{\prime \prime}(\xi)\right)}
$$

After putting values of $c_{1}$ and $c_{2}$, we get (2.7).

Theorem 2.7 enables us to define new mean. If we set

$$
H(\xi)=\frac{h^{2}(\xi) f^{\prime \prime}(\xi)-2 h(\xi) h^{\prime}(\xi) f^{\prime}(\xi)+f(\xi)\left(2 h^{\prime}(\xi)-h(\xi) h^{\prime \prime}(\xi)\right)}{h^{2}(\xi) g^{\prime \prime}(\xi)-2 h(\xi) h^{\prime}(\xi) g^{\prime}(\xi)+g(\xi)\left(2 h^{\prime}(\xi)-h(\xi) h^{\prime \prime}(\xi)\right)}
$$

and suppose that $H$ is invertible, then

$$
\xi=H^{-1}\left(\frac{D_{n, f}(\mathbf{x}, \mathbf{y} ; \mathbf{p})}{D_{n, g}(\mathbf{x}, \mathbf{y} ; \mathbf{p})}\right)
$$

is a new mean of two real numbers.

Corollary 2.9. If we put $h(x)=x$ and $p_{i}=1$ for $i=1, \ldots, n$ in (2.7) and (2.9), then we get [1, Theorem 2.4] and [1, Corollary 2.7] respectively. 
Lemma 2.10. Let $r \in \mathbf{R}$ and $\psi_{r}:(0, \infty) \rightarrow \mathbf{R}$ be the class of functions defined as

$$
\psi_{r}(x)= \begin{cases}\frac{x^{r} h(x)}{r(r-1)}, & r \neq 0,1 \\ x h(x) \log x, & r=1 \\ -h(x) \log x, & r=0\end{cases}
$$

Then $\frac{\psi_{r}}{h}$ is convex on $(0, \infty)$ for each $r \in \mathbf{R}$.

Proof. Since

$$
\left(\frac{\psi_{r}(x)}{h(x)}\right)^{\prime \prime}=x^{r-2}>0, \forall x \in(0, \infty) \text { and } r \in \mathbf{R},
$$

therefore the given functions are convex on $(0, \infty)$.

Using above class of functions in Theorem 2.6, we get the following result.

Let $\psi_{r}$ with assumptions in Theorem 2.6, we have

$$
\frac{D_{n, \psi_{p}}(\mathbf{x}, \mathbf{y} ; \mathbf{p})}{D_{n, \psi_{r}}(\mathbf{x}, \mathbf{y} ; \mathbf{p})}=\xi^{p-r}, \quad p \neq r
$$

for some $\xi \in I \subset(0, \infty)$ such that $D_{n, \psi_{s}}(\mathbf{x}, \mathbf{y}, \mathbf{p}) \neq 0, s=p, r$ and so we have another mean

$$
\xi=\left(\frac{D_{n, \psi_{p}}(\mathbf{x}, \mathbf{y} ; \mathbf{p})}{D_{n, \psi_{r}}(\mathbf{x}, \mathbf{y} ; \mathbf{p})}\right)^{\frac{1}{p-r}} .
$$

\section{3. m-Exponential convexity, Cauchy means and their mono- tonicity}

We start this section by giving some definitions and notions which are used frequently in the results (see [10]). Throughout this section $I$ is an interval in $\mathbf{R}$.

Definition 3.1. A function $f: I \rightarrow \mathbf{R}$ is $m$-exponentially convex in the Jensen sense on $I$, if

$$
\sum_{i, j=1}^{m} \xi_{i} \xi_{j} f\left(\frac{x_{i}+x_{j}}{2}\right) \geq 0
$$

holds for all choices $\xi_{i} \in \mathbf{R}$ and $x_{i} \in I, i=1, \ldots, m$.

A function $f: I \longrightarrow \mathbf{R}$ is $m$-exponentially convex if it is $m$-exponentially convex in the Jensen sense and continuous on $I$. 
Remark 3.2. It is clear from the definition that 1-exponentially convex functions in the Jensen sense are in fact nonnegative functions. Also, $\mathrm{m}$ exponentially convex functions in the Jensen sense are $k$-exponentially convex in the Jensen sense for every $k \in \mathbf{N}, k \leq m$.

By using some linear algebra and definition of positive semi-definite matrices, we have the following proposition.

Proposition 3.3. If $f$ is an $m$-exponentially convex function in the Jensen sense then the matrix

$$
\left[f\left(\frac{x_{i}+x_{j}}{2}\right)\right]_{i, j=1}^{k}
$$

is a positive semi-definite matrix for all $k \in \mathbf{N}, k \leq m$. In particular,

$$
\operatorname{det}\left[f\left(\frac{x_{i}+x_{j}}{2}\right)\right]_{i, j=1}^{k} \geq 0
$$

for all $k \in \mathbf{N}, k \leq m$.

Definition 3.4. A function $f: I \rightarrow \mathbf{R}$ is exponentially convex in the Jensen sense on $I$ if it is $m$-exponentially convex in the Jensen sense for all $m \in \mathbf{N}$.

A function $f: I \rightarrow \mathbf{R}$ is exponentially convex if it is exponentially convex in the Jensen sense and continuous on $I$.

Lemma 3.5. It is known (and easy to show) that $f: I \rightarrow \mathbf{R}^{+}$is log-convex in the Jensen sense if and only if

$$
l^{2} f(t)+2 \operatorname{lm} f\left(\frac{t+r}{2}\right)+m^{2} f(r) \geq 0
$$

holds for each $l, m \in \mathbf{R}$ and $r, t \in I$.

It follows that a positive function is log-convex in the Jensen sense if and only if it is 2-exponentially convex in the Jensen sense. Also, using basic convexity theory it follows that a positive function is log-convex if and only if it is 2-exponentially convex.

The following lemma is equivalent to the definition of convex function [12, page 2].

Lemma 3.6. If $x_{1}, x_{2}, x_{3} \in I$ are such that $x_{1}<x_{2}<x_{3}$, then the function $f: I \rightarrow \mathbf{R}$ is convex if and only if inequality

$$
\left(x_{3}-x_{2}\right) f\left(x_{1}\right)+\left(x_{1}-x_{3}\right) f\left(x_{2}\right)+\left(x_{2}-x_{1}\right) f\left(x_{3}\right) \geq 0
$$

holds. 
Theorem 3.7. Let $\mathbf{x}$ and $\mathbf{y}$ be positive $n$-tuples, $\mathbf{p} \in(0,1]^{n}$ and $\psi_{r}$ be a function defined in Lemma 2.10. Then $r \mapsto D_{n, \psi_{r}}(\mathbf{x}, \mathbf{y} ; \mathbf{p})$ is $m$-exponentially convex.

Proof. Consider the function $f$ define as

$$
f(x)=\sum_{i, j=1}^{m} u_{i} u_{j} \psi_{r_{i j}}(x)
$$

for $x>0, u_{i} \in \mathbf{R}$ and $r_{i j}=\frac{r_{i}+r_{j}}{2}$. Then

$$
\begin{gathered}
\frac{f(x)}{h(x)}=\sum_{i, j=1}^{m} u_{i} u_{j} \frac{\psi_{r_{i j}}(x)}{h(x)} \\
\left(\frac{f(x)}{h(x)}\right)^{\prime \prime}=\sum_{i, j=1}^{m} u_{i} u_{j} x^{r_{i j}-2}=\left(\sum_{i=1}^{m} u_{i} x^{\frac{r_{i}-1}{2}}\right)^{2} \geq 0, \forall x>0 .
\end{gathered}
$$

that is $\frac{f(x)}{h(x)}$ is convex function for all $x>0$, using $f(x)$ defined above in (2.2) we have

$\sum_{i=1}^{n} 4\left\{\frac{1}{2}\left(f\left(x_{i}\right)+f\left(y_{i}\right)\right)-f\left(\frac{x_{i}+y_{i}}{2}\right)\right\}-p_{i}\left(h\left(x_{i}\right)-h\left(y_{i}\right)\right)\left(\frac{f\left(x_{i}\right)}{h\left(x_{i}\right)}-\frac{f\left(y_{i}\right)}{h\left(y_{i}\right)}\right) \geq 0$,

using value of $f(x)$ we obtain

$$
\sum_{i, j=1}^{m} u_{i} u_{j} D_{n, \psi_{t_{i j}}}(\mathbf{x}, \mathbf{y} ; \mathbf{p}) \geq 0 \text {. }
$$

This implies $r \mapsto D_{n, \psi_{r}}(\mathbf{x}, \mathbf{y} ; \mathbf{p})$ is $n$-exponentially convex in Jensen sense. Also we have $\lim _{r \rightarrow 0} D_{n, \psi_{r}}(\mathbf{x}, \mathbf{y} ; \mathbf{p})=D_{n, \psi_{0}}(\mathbf{x}, \mathbf{y} ; \mathbf{p})$ and

$\lim _{r \rightarrow 1} D_{n, \psi_{r}}(\mathbf{x}, \mathbf{y} ; \mathbf{p})=D_{n, \psi_{1}}(\mathbf{x}, \mathbf{y} ; \mathbf{p})$, that is, $D_{n, \psi_{r}}(\mathbf{x}, \mathbf{y} ; \mathbf{p})$ is continuous for all $r \in \mathbf{R}$. This gives $r \mapsto D_{n, \psi_{r}}(\mathbf{x}, \mathbf{y} ; \mathbf{p})$ is $m$-exponentially convex.

Remark 3.8. It is also interesed to note that by setting $p_{i}=1, h_{i}(x)=$ $h(x)=x$ for $i=1, \ldots, n$ in above theorem, we obtain $m$-exponential convexity of $D$-divergence given in [4]. Log-convexity and exponential convexity of $D$-divergence proved in [4] followed from the following results.

Corollary 3.9. Let $\mathbf{x}$ and $\mathbf{y}$ be distinct positive $n$-tuples, $\mathbf{p} \in(0,1]^{n}$ and $\psi_{r}$ be a function defined in Lemma 2.10. Then $r \mapsto D_{n, \psi_{r}}(\mathbf{x}, \mathbf{y} ; \mathbf{p})$ is exponentially convex. 
Proof. It is an immediate consequence of the Theorem 3.7.

Corollary 3.10. Let $\mathbf{x}$ and $\mathbf{y}$ be distinct positive $n$-tuples, $\mathbf{p} \in(0,1]^{n}$ and $\psi_{r}$ be a function defined in Lemma 2.10. Then $r \mapsto D_{n, \psi_{r}}(\mathbf{x}, \mathbf{y} ; \mathbf{p})$ is 2 -exponentially convex and thus log-convex function. Also for $r, s, t \in \mathbf{R}$ such that $r<s<t$, we have

$$
\left[D_{n, \psi_{s}}(\mathbf{x}, \mathbf{y} ; \mathbf{p})\right]^{t-r} \leq\left[D_{n, \psi_{r}}(\mathbf{x}, \mathbf{y} ; \mathbf{p})\right]^{t-s}\left[D_{n, \psi_{t}}(\mathbf{x}, \mathbf{y} ; \mathbf{p})\right]^{s-r} .
$$

Proof. Since $\mathbf{x}$ and $\mathbf{y}$ be distinct positive n-tuples, therefore $D_{n, \psi_{r}}(\mathbf{x}, \mathbf{y} ; \mathbf{p})$ is positive. Also from Theorem 3.7, it is 2-exponentially convex and by Lemma 3.5, we have $r \mapsto D_{n, \psi_{r}}(\mathbf{x}, \mathbf{y} ; \mathbf{p})$ is log-convex.

Now for $r, s, t \in \mathbf{R}$ such that $r<s<t$ and $f(r)=D_{n, \psi_{r}}(\mathbf{x}, \mathbf{y} ; \mathbf{p})$ in Lemma 3.6, we have

$(t-r) \log D_{n, \psi_{s}}(\mathbf{x}, \mathbf{y} ; \mathbf{p}) \leq(t-s) \log D_{n, \psi_{r}}(\mathbf{x}, \mathbf{y} ; \mathbf{p})+(s-r) \log D_{n, \psi_{t}}(\mathbf{x}, \mathbf{y} ; \mathbf{p})$

This is equivalent to inequality (3.2).

Theorem 3.11. Let $\mathbf{x}$ and $\mathbf{y}$ be positive n-tuples, $\mathbf{p} \in(0,1]^{n}$ and $\psi_{r}$ be a function defined in Lemma 2.10. Then for $r_{i} \in \mathbf{R}, i=1, \ldots, m$, the matrix $A=\left[D_{n, \psi_{\frac{r_{i}+r_{j}}{2}}}(\mathbf{x}, \mathbf{y} ; \mathbf{p})\right]_{i, j=1}^{k}$ is a positive semi definite matrix. In particular

$$
\operatorname{det}\left[D_{n, \psi_{\frac{p_{i}+p_{j}}{2}}}(\mathbf{x}, \mathbf{y} ; \mathbf{p})\right]_{i, j=1}^{k} \geq 0 \forall k=1, \ldots, m
$$

Proof. Since $r \mapsto D_{n, \psi_{r}}(\mathbf{x}, \mathbf{y} ; \mathbf{p})$ is $n$-exponentially convex function, therefore by Proposition 3.3, we have the required result.

Definition 3.12. Let $\mathbf{x}$ and $\mathbf{y}$ be two positive n-tuples. For $r, s \in \mathbf{R}$ we define generalized mean $M_{s, r}(\mathbf{x}, \mathbf{y})$ defined as

$$
M_{s, r}=\left(\frac{D_{n, \psi_{s}}(\mathbf{x}, \mathbf{y} ; \mathbf{p})}{D_{n, \psi_{r}}(\mathbf{x}, \mathbf{y} ; \mathbf{p})}\right)^{\frac{1}{s-r}}, \quad s \neq r
$$

where $D_{n, \psi_{p}}(\mathbf{x}, \mathbf{y} ; \mathbf{p}) \neq 0$ for $p=r, s$.

Other cases can be obtained by taking limits as: $\mathrm{M}_{s, 0}(\mathbf{X}, \mathbf{y})=$ 
$\left\{\frac{-(s(s-1))^{-1} \sum_{i=1}^{n}\left\{4\left(\frac{x_{i}+y_{i}}{2}\right)^{s+1} \cdot h\left(\frac{x_{i}+y_{i}}{2}\right)-2\left(x_{i}^{s+1} \cdot h\left(x_{i}\right)+y_{i}^{s+1} \cdot h\left(y_{i}\right)\right)+p_{i}\left(h\left(x_{i}\right)-\left(y_{i}\right)\right)\left(x_{i}^{s}-y_{i}^{s}\right)\right\}}{\sum_{s \neq 0,1}^{n}\left\{2\left(x_{i}+y_{i}\right) \log \frac{x_{i}+y_{i}}{2} \cdot h\left(\frac{x_{i}+y_{i}}{2}\right)-2\left(x_{i} \log x_{i} \cdot h\left(x_{i}\right)+y_{i} \log y_{i} \cdot h\left(y_{i}\right)\right)+p_{i}\left(h\left(x_{i}\right)-h\left(y_{i}\right)\right)\left(\log x_{i}-\log y_{i}\right)\right\}}\right\}^{\frac{1}{s}}$, $\mathrm{M}_{1,0}(\mathbf{X}, \mathbf{Y})=$

$\left\{\frac{-\sum_{i=1}^{n}\left\{\left(x_{i}+y_{i}\right)^{2} \log \left(\frac{x_{i}+y_{i}}{2}\right) \cdot h\left(\frac{x_{i}+y_{i}}{2}\right)-2\left(x_{i}^{2} \log x_{i} \cdot h\left(x_{i}\right)+y_{i}^{2} \log y_{i} \cdot h\left(y_{i}\right)\right)+\left(h\left(x_{i}\right)-h\left(y_{i}\right)\right)\left(x_{i} \log x_{i}-y_{i} \log y_{i}\right)\right\}}{\sum_{\mathbf{M}_{s, s}(\mathbf{X}, 1}^{n}\left\{2\left(x_{i}+y_{i}\right) \log \frac{x_{i}+y_{i}}{2} \cdot h\left(\frac{x_{i}+y_{i}}{2}\right)-2\left(x_{i} \log x_{i} \cdot h\left(x_{i}\right)+y_{i} \log y_{i} \cdot h\left(y_{i}\right)\right)+\left(h\left(x_{i}\right)-h\left(y_{i}\right)\right)\left(\log x_{i}-\log y_{i}\right)\right\}}\right\}$,

$\left\{\frac{\sum_{i=1}^{n}\left\{4\left(\frac{x_{i}+y_{i}}{2}\right)^{s+1} \log \left(\frac{x_{i}+y_{i}}{2}\right) \cdot h\left(\frac{x_{i}+y_{i}}{2}\right)-2\left(x_{i}^{s+1} \log x_{i} \cdot h\left(x_{i}\right)+y_{i}^{s+1} \log y_{i} \cdot h\left(y_{i}\right)\right)+\left(h\left(x_{i}\right)-h\left(y_{i}\right)\right)\left(x_{i}^{s} \log x_{i}-y_{i}^{s} \log y_{i}\right)\right\}}{\sum_{i=1}^{n}\left\{4\left(\frac{x_{i}+y_{i}}{2}\right)^{s+1} h\left(\frac{x_{i}+y_{i}}{2}\right)-2\left(x_{i}^{s+1} h\left(x_{i}\right)+y_{i}^{s+1} \cdot h\left(y_{i}\right)\right)+h\left(\left(x_{i}\right)-h\left(y_{i}\right)\right)\left(x_{i}^{s}-y_{i}^{s}\right)\right\}}\right.$

$\left.-\frac{2 s-1}{s(s-1)}\right\}, \quad s \neq 0,1$ $\mathrm{M}_{0,0}(\mathbf{X}, \mathbf{Y})=\exp$

$\left\{\frac{\sum_{i=1}^{n}\left\{2\left(x_{i}+y_{i}\right) \log ^{2}\left(\frac{x_{i}+y_{i}}{2}\right) \cdot h\left(\frac{x_{i}+y_{i}}{2}\right)-2\left(x_{i} \log ^{2} x_{i} \cdot h\left(x_{i}\right)+y_{i} \log ^{2} y_{i} \cdot h\left(y_{i}\right)\right)+\left(h\left(x_{i}\right)-h\left(y_{i}\right)\right)\left(\log ^{2} x_{i}-\log ^{2} y_{i}\right)\right\}}{2 \sum_{i=1}^{n}\left\{2\left(x_{i}+y_{i}\right) \log \left(\frac{x_{i}+y_{i}}{2}\right) \cdot h\left(\frac{x_{i}+y_{i}}{2}\right)-2\left(x_{i} \log x_{i} \cdot h\left(x_{i}\right)+y_{i} \log y_{i} \cdot h\left(y_{i}\right)\right)+\left(h\left(x_{i}\right)-h\left(y_{i}\right)\right)\left(\log x_{i}-\log y_{i}\right)\right\}}+1\right\}$ $\mathrm{M}_{1,1}(\mathbf{X}, \mathbf{Y})=\exp$

$\left\{\frac{\sum_{i=1}^{n}\left\{\left(x_{i}+y_{i}\right)^{2} \log ^{2}\left(\frac{x_{i}+y_{i}}{2}\right) \cdot h\left(\frac{x_{i}+y_{i}}{2}\right)-2\left(x_{i}^{2} \log ^{2} x_{i} \cdot h\left(x_{i}\right)+y_{i}^{2} \log ^{2} y_{i} \cdot h\left(y_{i}\right)\right)+\left(h\left(x_{i}\right)-h\left(y_{i}\right)\right)\left(x_{i} \log ^{2} x_{i}-y_{i} \log ^{2} y_{i}\right)\right\}}{2 \sum_{i=1}^{n}\left\{\left(x_{i}+y_{i}\right)^{2} \log \left(\frac{x_{i}+y_{i}}{2}\right) \cdot h\left(\frac{x_{i}+y_{i}}{2}\right)-2\left(x_{i}^{2} \log x_{i} \cdot h\left(x_{i}\right)+y_{i}^{2} \log y_{i} \cdot h\left(y_{i}\right)\right)+\left(h\left(x_{i}\right)-h\left(y_{i}\right)\right)\left(x_{i} \log x_{i}-y_{i} \log y_{i}\right)\right\}}+1\right\}$

Theorem 3.13. For $p, r, s, t \in \mathbf{R}$ such that $r \leq s$ and $p \leq t$ we have

$$
M_{p, r}(\mathbf{x}, \mathbf{y}) \leq M_{t, s}(\mathbf{x}, \mathbf{y}) \text {. }
$$

Proof. The following inequality holds for convex function $\varphi$ see in $[12$, p. 4],

$$
\frac{\varphi\left(x_{2}\right)-\varphi\left(x_{1}\right)}{x_{2}-x_{1}} \leq \frac{\varphi\left(y_{2}\right)-\varphi\left(y_{1}\right)}{y_{2}-y_{1}}
$$

where $x_{1} \leq y_{1}, x_{2} \leq y_{2}, x_{1} \neq x_{2}, y_{1} \neq y_{2}$.

Since $r \mapsto D_{n, \psi_{r}}(\mathbf{x}, \mathbf{y} ; \mathbf{r})$ is log-convex, we can put in (3.4): $\varphi(r)=\log D_{n, \psi_{r}}(\mathbf{x}, \mathbf{y} ; \mathbf{p}), x_{1}=p, x_{2}=r, y_{1}=t, y_{2}=s$. We get for $p \neq$ $r, t \neq s$

$\frac{\log D_{n, \psi_{p}}(\mathbf{x}, \mathbf{y} ; \mathbf{p})-\log D_{n, \psi_{r}}(\mathbf{x}, \mathbf{y} ; \mathbf{p})}{p-r} \leq \frac{\log D_{n, \psi_{t}}(\mathbf{x}, \mathbf{y} ; \mathbf{p})-\log D_{n, \psi_{s}}(\mathbf{x}, \mathbf{y} ; \mathbf{p})}{t-s}$

therefore we have

$$
\left(\frac{D_{n, \psi_{p}}(\mathbf{x}, \mathbf{y} ; \mathbf{p})}{D_{n, \psi_{r}}(\mathbf{x}, \mathbf{y} ; \mathbf{p})}\right)^{\frac{1}{p-r}} \leq\left(\frac{D_{n, \psi_{t}}(\mathbf{x}, \mathbf{y} ; \mathbf{p})}{D_{n, \psi_{s}}(\mathbf{x}, \mathbf{y} ; \mathbf{p})}\right)^{\frac{1}{t-s}}
$$

From (3.5) we get our result for $p \neq r, t \neq s$ and for $p=r, t=s ; p \neq$ $r, t=s ; p=r, t \neq s$ we can consider limiting cases.

Remark 3.14. If we put $h(x)=x$ and $p_{i}=1$ for $i=1, \ldots, n$ in above means, then we get means defined in [1] and above theorem gives [1, Theorem 2.11] respectively. 


\section{References}

[1] M. Anwar, G. Farid and J. Pečarić, Generalization of K-divergence and related results, J. Math. Ineq., 5(2), pp. 181-191, (2011).

[2] L. Boltzman, Neitere Studien fiber das Warmegleichgewicht unter Gasmolekulen, K. Akad. Wiss. (Wein) Sitzb. 66, 275, (1872).

[3] J. Burbea, and C.R. Rao, Entropy differential metric, distance and divergence measures in probability spaces: A unified approach, J. Multivariate Anal. 12(4), pp. 575-596, (1982).

[4] J. Burbea and C. R. Rao, On convexity of some divergence measure based on entropy functions, IEEE Trans. Inform. Theory 28(3), pp. 489-495, (1982).

[5] R. Clausius, Abhaudlungen fiber die mechanische Wiirmetheorie Friedrich Vieweg, Braunschweig, (1864).

[6] R. G. Gallager, Information theory and reliable communication, Willy, New York, (1968).

[7] S. Hussain, On Jensen's and related inequalities, $\mathrm{PhD}$ thesis, Govt. College University, Lahore, $2009 . \quad$ URL: http://eprints.hec.gov.pk/6348/.

[8] R. C. Lewonton, The apportionment of human diversity, Evol. Biol. 6, pp. 381-398, (1972).

[9] P. C. Mahalanobis, On the generalized distance in statistics, Proc. Natl. Inst. Sci. 2(1), pp. 49-55, (1936).

[10] J. Pečarić and J. Perić, Improvements of the Giaccardi and the Petrovic inequality and related Stolarsky type means, An. Univ. Craiova Ser. Mat. Inform., 39(1), pp. 65-75, (2012).

[11] M. A. Noor, K. I. Noor and M. U. Awan, Geometrically relative convex functions, Appl. Math. Inf. Sci. 8(2), pp. 607-616, (2014).

[12] J. E. Pečarić, F. Proschan and Y. L. Tong, Convex functions, partial orderings and statistical applications, Academic Press, New York, (1992). 
[13] E. C. Pielou, Ecological diversity, Willy, New York, (1975).

[14] C. R. Rao, Diversity and dissimilarity coefficients, Theor. Pop. Bio. 21, pp. 24-43, (1982).

[15] C. E. Shannon, A mathematical theory of communication, Bell Syst. Tech. J. 27, pp. 379-423 and pp. 623-656, (1948).

[16] A. Ullah, Entropy, divergence and distance measures with econometric applications, J Stat Plan Inference. 49, pp. 137-162, (1996).

[17] N. Wiener, Cybernetics or control and communication in the animal and the machine, 2nd Edition, The MIT Press, (1965).

\author{
G. Farid \\ COMSATS Institute of Information Technology, \\ Attock Campus, \\ Pakistan \\ e-mail : ghlmfarid@ciit-attock.edu.pk

\section{Atiq Ur Rehman} \\ COMSATS Institute of Information Technology, \\ Attock Campus, \\ Pakistan \\ e-mail : atiq@mathcity.org \\ and

\section{J. Pečarić} \\ Faculty Of Textile Technology Zagreb, \\ University Of Zagreb, \\ Croatia \\ e-mailpecaric@mahazu.hazu.hr
}

